\title{
COMPLEX STRUCTURES ON REAL BANACH SPACES
}

\section{JEAN DIEUDONNE}

1. Let $E_{0}$ be a topological vector space over the complex number field $\boldsymbol{C}$. The mapping $(\zeta, x) \rightarrow \zeta x$ is then continuous when one restricts $\zeta$ to take only real values. Hence, we have on $E_{0}$ a structure of topological vector space over the real number field $R$. We shall denote by $E$ that topological vector space. The homothetic mapping $x \rightarrow i x$ of $E_{0}$ onto itself is an automorphism $u$ of the topological vector space $E$, such that $u^{2}(x)=-x$. Conversely, let $E$ be a topological vector space over $R$, and let $u$ be an automorphism of $E$ such that $u^{2}(x)=-x$. One can then define on $E$ a structure of vector space over $C$, by setting $(\lambda+i \mu) x=\lambda x+\mu u(x)$. The axioms of vector spaces are trivially verified, and the continuity of $u$ insures that the mapping $(\zeta, x) \rightarrow \zeta x$ of $C \times E$ into $E$ is continous. One defines thus a topological vector space $E_{0}$ over $C$, from which the original space $E$ can be derived as above.

When a topological vector space $E$ over $R$ is given, the question naturally arises of the existence of an automorphism $u$ of $E$ such that $u^{2}(x)=-x$. It is well known that when $E$ has finite dimension $n$, the necessary and sufficient condition for the existence of $u$ is that $n$ be an even number. In this note, we shall give an example of an infinite-dimensional Banach space $E$ over $R$, such that there exists no automorphism $u$ of $E$ with the required property.

2. When $E$ is a Banach space over $R$, and an automorphism $u$ of $E$ such that $u^{2}(x)=-x$ exists, the topology of the space $E_{0}$ (which is identical with the topology of $E$ ) can still be defined by a norm, for instance $\|x\|_{0}=\sup _{0 \leq 0 \leq 2 \pi}\left\|e^{i \theta} x\right\|(\|x\|$ meaning the norm on $E)$; for one has obviously $\|x\| \leqq\|x\|_{0} \leqq(1+\|u\|)\|x\|$. Let $E^{\prime}$ and $E_{0}^{\prime}$ be the $d u a l$ spaces of $E$ and $E_{0}$ respectively. $E^{\prime}$ is a Banach space over $\boldsymbol{R}$ and $E_{0}^{\prime}$ a Banach space over $C$. There is a well known natural mapping of $E^{\prime}$ onto $E_{0}^{\prime}$ which to every continuous linear form $v \in E^{\prime}$ associates the continuous linear form $w=\phi(v)$ over $E_{0}$ such that $w(x)=v(x)-i v(i x)$. The inverse mapping $v=\psi(w)$ is such that $v(x)$ $=\Re(w(x))$. As $|w(x)| \leqq(1+\|u\|)\|v\| \cdot\|x\|$, and $|v(x)| \leqq|w(x)|$, it is clear that $\phi$ and $\psi$ are continuous; $\phi$ is therefore an isomorphism of the topological vector space $E^{\prime}$ over $R$ onto the topological vector space $E_{0}^{\prime}$ over $R$.

Now let $E^{\prime \prime}$ be the dual of the Banach space $E^{\prime}$ (over $R$ ). Let $E_{0}^{\prime \prime}$

Received by the editors March 5, 1951. 
be the dual of the Banach space $E_{0}^{\prime}$, when $E_{0}^{\prime}$ is considered as a space over $R$; and let $E_{00}^{\prime \prime}$ be the dual of the Banach space $E_{0}^{\prime}$, when $E_{0}^{\prime}$ is considered as a space over $C$. The same argument as before yields a natural mapping $\phi^{\prime}$ of $E_{0}^{\prime \prime}$ onto $E_{00}^{\prime \prime}$, which is an isomorphism for the structures of topological vector spaces over $\boldsymbol{R}$ of these spaces. Moreover, the mapping which to any continuous linear form $V$ over $E^{\prime}$ associates the continuous linear form $w \rightarrow V\left(\psi(w)\right.$ ) over $E_{0}^{\prime}$ (considered as a space over $R$ ) is again an isomorphism of $E^{\prime \prime}$ onto $E_{0}^{\prime \prime}$ (for the structures of topological spaces over $R$ ). We thus get finally a natural isomorphism $\Phi$ of $E^{\prime \prime}$ onto $E_{00}^{\prime \prime}$, when both these spaces are considered as topological vector spaces over $\boldsymbol{R}$.

Moreover, there is a natural isomorphism $x \rightarrow U_{x}$ of the Banach space $E$ into the Banach space $E^{\prime \prime}$, such that $U_{x}(v)=v(x)$ for every $v \in E^{\prime}$. Similarly, there is a natural isomorphism $x \rightarrow U_{x}^{0}$ of the Banach space $E_{0}$ (over $\boldsymbol{C}$ ) into the Banach space $E_{00}^{\prime \prime}$ (over $\boldsymbol{C}$ ). For every $x \in E_{0}$, and every $w \in E_{0}^{\prime}$, one has $U_{x}(\psi(w))=\Re(w(x))$, and therefore, if $T(w)=U_{x}(\psi(w))$, and $W=\phi^{\prime}(T)$, one has $W(w)=\Re(w(x))$ $-i \Re(w(i x))=w(x)=U_{x}^{0}(w)$. In other words, $\Phi\left(U_{x}\right)=U_{x}^{0}$ for every $x \in E$, which means that under the isomorphism $\Phi, E$ (considered as imbedded in $E^{\prime \prime}$ ) is transformed into $E_{0}$ (considered as imbedded in $\left.E_{00}^{\prime \prime}\right)$.

3. Now R. C. James has given recently an example of a Banach space $E$ over $R$, such that $E^{\prime \prime} / E$ has dimension one over $R .{ }^{1}$ Suppose there existed an automorphism $u$ of $E$ such that $u^{2}(x)=-x$; it would define a Banach space $E_{0}$ over $C$, and it follows from $\$ 2$ that $E_{00}^{\prime \prime} / E_{0}$ would have dimension one over $R$. But $E_{00}^{\prime \prime} / E_{0}$ is a vector space over $C$ which is not reduced to 0 , and as such its dimension over $R$ is at least 2 . We thus reach a contradiction, which proves our contention.

The same example exhibits another interesting feature concerning the problem we are considering. Namely, there exists an increasing sequence $\left(L_{n}\right)$ of closed subspaces of $E$, whose union $M$ is dense in $E$, and a (noncontinuous) one-to-one linear mapping $u$ of $M$ onto itself, such that $u^{2}(x)=-x$, and that $u$, restricted to any one of the $L_{n}$, is a (continuous) automorphism of that subspace. In fact, it is known ${ }^{1}$ that there exists in $E$ two closed subspaces $H_{1}, H_{2}$, each of which is (as a topological vector space) isomorphic to a separable Hilbert space, and such that $H_{1} \cap H_{2}=\{0\}$, and that $M=H_{1}+H_{2}$ is dense in $E$. If $\left(a_{n}\right)$ and $\left(b_{n}\right)$ are orthogonal bases of $H_{1}$ and $H_{2}$ respectively, $u$ is defined by taking $u\left(a_{2 n-1}\right)=a_{2 n}, u\left(a_{2 n}\right)=-a_{2 n-1}, u\left(b_{2 n-1}\right)=b_{2 n}$,

${ }^{1}$ R. C. James, Bases and reflexivity of Banach spaces, Ann. of Math. vol. 52 (1950) pp. 518-527. 
$u\left(b_{2 n}\right)=-b_{2 n-1}$; and $L_{n}$ is the closed subspace of $E$ generated by $H_{1}$ and the vectors $b_{i}$ such that $1 \leqq i \leqq 2 n$.

Such a situation excludes the possibility of proving the existence of a complex structure on a real Banach space by an inductive argument (of the type used, for instance, in the proof of Hahn-Banach's theorem). As was pointed out by the referee, the same example shows that a linear mapping of $M$ onto itself can be continuous on both subspaces $H_{1}, H_{2}$ without being continuous on $M$ itself.

The Johns Hopkins University 